\title{
Relationship between the Content of Lysyl Oxidase-dependent Cross-Links in Skin Collagen, Nonenzymatic Glycosylation, and Long-Term Complications in Type I Diabetes Mellitus
}

Bruce Buckingham* and Karen M. Reiser*

*Department of Pediatric Endocrinology, Children's Hospital of Orange County, Orange, California 92668; and

${ }^{\ddagger}$ Department of Internal Medicine, School of Medicine, University of California, Davis, California 95616

\begin{abstract}
Many abnormalities in collagen have been reported in insulindependent diabetes mellitus, some or all of which have been attributed to increased cross-linking. Although recent work has focused on the role of glucose-derived collagen cross-links in the pathogenesis of diabetic complications, relatively few studies have investigated the role of lysyl oxidase-dependent (LOX) cross-links. In the present study, LOX cross-links and nonenzymatic glycosylation were quantified in skin collagen from diabetic subjects. There was an increase in the difunctional cross-link dihydroxylysinonorleucine (DHLNL) as well as in one of its trifunctional maturation products, hydroxypyridinium. All other LOX crosslinks were normal. Nonenzymatic glycosylation was increased in diabetic skin collagen, and this increase was correlated with increases in DHLNL $(P<0.001)$. The biochemical results were examined for correlations with clinical data from the same subjects. Increases in DHLNL content were associated with duration of diabetes $(P<0.003)$, glycohemoglobin levels $(P<0.001)$, hand contractures $(P$ $<0.05)$, skin changes $(P<0.005)$, and microalbuminuria $(P$ $<0.01)$. In nondiabetic subjects age was not correlated with collagen cross-link content with the exception that his-HLNL increased with age $(r=0.79, P<0.02)$. In diabetic subjects, PA levels decreased with age $(r=0.51, P<0.02)$. With increased duration of diabetes, DHLNL content was increased ( $r$ $=0.55, P<0.003)$ and OHP was increased $(r=0.59, P$ $<0.01)$, whereas PA levels were decreased $(r=-0.48, P$ $<0.04$ ) (Fig. 5). Nonenzymatic glycosylation of collagen was also increased with increased duration of diabetes (hex-lys, $r$ $=0.47, P<0.02$; hex-hyl, $r=0.39, P<0.05)$. We conclude that: (a) lysyl oxidase-dependent cross-linking is increased in skin collagen in diabetes and (b) that these changes in skin collagen are correlated with duration of diabetes, glycemic control, and long-term complications. (J. Clin. Invest. 1990. 86:1046-1054.) Key words: collagen • cross-links • diabetes • nonenzymatic glycosylation
\end{abstract}

A preliminary report of this study was presented at the June, 1988, meeting of the American Diabetes Association.

Address reprint requests to Dr. Karen M. Reiser, CPRC, University of California, Davis, Davis, CA 95616.

Received for publication 20 October 1988 and in revised form 9 May 1990

J. Clin. Invest.

(c) The American Society for Clinical Investigation, Inc. 0021-9738/90/10/1046/09 \$2.00

Volume 86, October 1990, 1046-1054

\section{Introduction}

There is considerable evidence suggesting that widespread derangements in collagen metabolism occur in insulin-dependent diabetes mellitus (IDDM). ${ }^{1}$ Collagen from many tissues and organs in diabetic patients, as well as in experimental diabetes, has long been reported to be more insoluble and more resistant to enzymatic digestion than normal collagen (1-3). Increased deposition of type IV collagen in capillary basement membranes may contribute, in part, to vascular complications such as retinopathy and nephropathy (4). In the syndrome of limited joint mobility, waxy skin, and short stature first described by Rosenbloom et al. (5), increased deposition of type I collagen in the dermis has been reported (6). It has been suggested that many of the observed abnormalities of collagen in IDDM may be attributed to increased cross-linking. What might be the nature of such crosslinks? Present knowledge suggests that there are at least two main pathways by which collagen molecules become progressively crosslinked. One pathway (Fig. $1 A$ ) begins with nonenzymatic glycosylation of lysine and hydroxylysine residues. These early glycosylation products are believed to undergo a series of reactions to form complex fluorophores and chromophores collectively referred to as advanced Maillard products or advanced glycosylation end-products (7). Several studies have shown that early glycosylation products are increased in collagen in both human and experimental diabetes (8). Advanced Maillard products are also believed to be increased in diabetic collagen $(7,9)$.

A second cross-link pathway (Fig. $1 B$ ) begins with the lysyl oxidase-dependent oxidative deamination of certain lysine and hydroxylysine residues. The resultant aldehyde moieties may undergo further reactions with lysine, hydroxylysine, and histidine residues to form di-, tri- and tetrafunctional crosslinks (10). There is evidence that lysyl oxidase-dependent crosslinking is increased in experimental diabetes (11). To our knowledge, no such studies have been done on tissue from patients with IDDM.

In the present study, we have used highly specific HPLC techniques to quantify both nonenzymatic glycosylation products and lysyl oxidase-dependent crosslinks in skin collagen biopsies obtained from patients with IDDM in order to test the following hypotheses: $(a)$ that lysyl oxidase-dependent crosslinks are increased in collagen in IDDM; $(b)$ that nonenzymatic glycosylation of collagen may affect the formation of spe-

1. Abbreviations used in this paper: DHLNL, dihydroxylysinornorleucine; hex-hyl, hexosyl-hydroxylysine; hex-lys, hexosyl lysine; HHMD, histidinohydroxymerodesmosine; HLNL, hydroxylysinonorleucine; IDDM, insulin-dependent diabetes mellitus; LP, lysyl pyridinium; OHP, hydroxypyridinium; PA, pyridinoline analogue. 


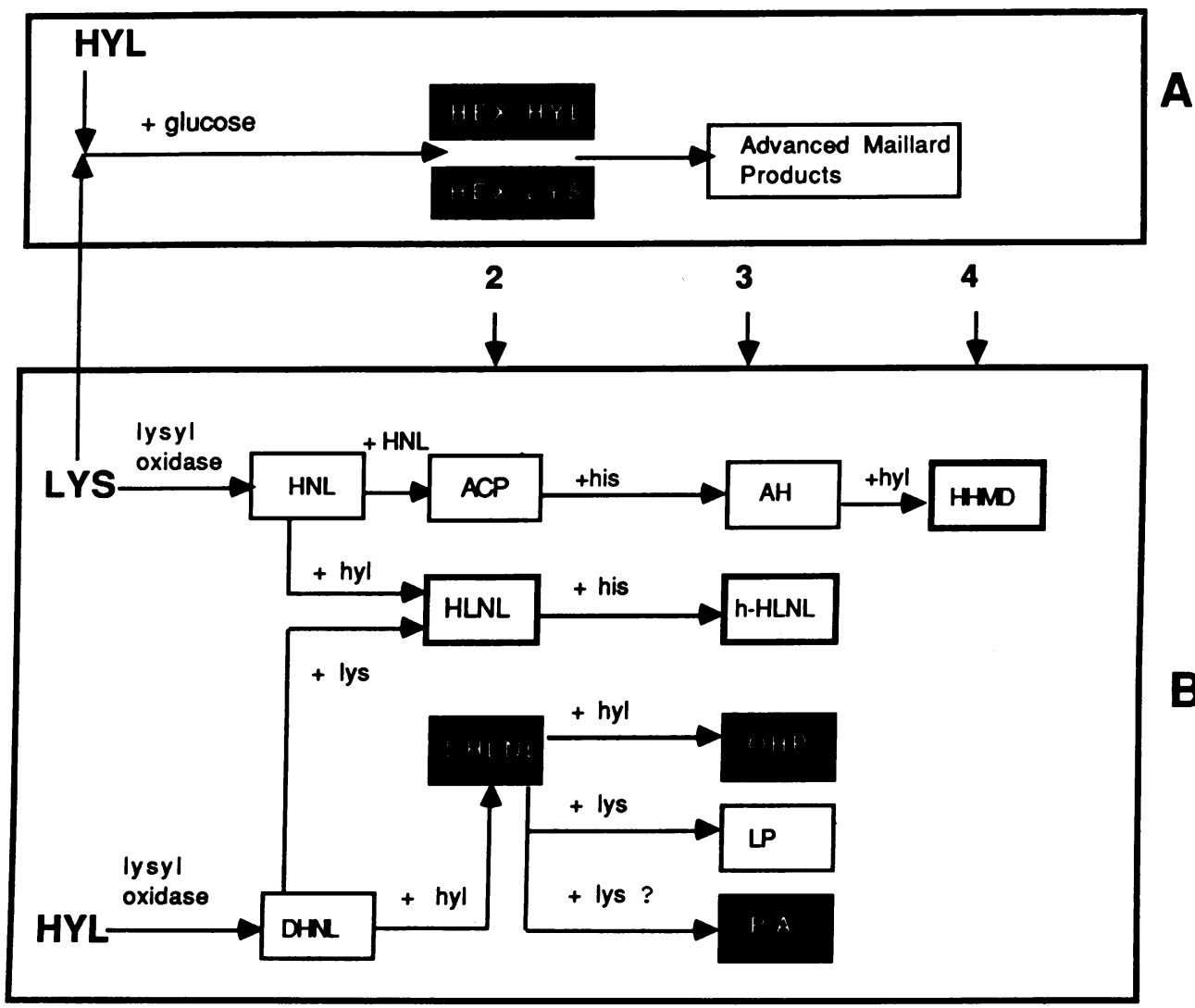

Figure 1. Reactions of collagenbound lysine and hydroxylysine, illustrating $(A)$ nonenzymatic glycosylation, which results in formation of hexosyl-lysine and hexosyl-hydroxylysine; some of these early difunctional products (the Schiff bases and their Amadori rearrangement products) may mature to advanced Maillard products. (B) Lysyl oxidasedependent reactions, showing progression from aldehyde moieties (HNL and DHNL) to ditri-, and tetrafunctional crosslinks. The numbers of residues are indicated by arrows at the top of $B$. Compounds in boldfaced boxes were analyzed in this study; compounds in solid black boxes were increased in diabetic skin collagen in this study.

cific lysyl oxidase-dependent cross-links; and $(c)$ that alterations in lysyl oxidase-dependent cross-links would be correlated with such clinical findings as glycemic control, duration of diabetes and long-term cutaneous and vascular complications.

\section{Methods}

Patient population. 26 patients with IDDM participated in this study after signing an informed consent form approved by the Children's Hospital of Orange County Human Subjects Review Committee (the institutional review board on human experimentation). There were 8 males and 18 females with a mean age of $16 \mathrm{yr}$ (range; 7-29 yr) and a mean duration of diabetes of $9.5 \mathrm{yr}$ (range: 1-16.5 yr). A 3-mm Baker's punch skin biopsy was obtained from the upper thigh after local anesthesia with $2 \%$ xylocaine with epinephrine. Nine control skin biopsies were obtained from nondiabetic subjects (two at autopsy, two at the time of surgical procedure, and five were punch biopsies after subjects gave informed consent). There were five male and four female controls with a mean age of $16 \mathrm{yr}$ (range: 7-29 yr).

Clinical studies. At the time of initial biopsy each patient was examined by an ophthalmologist specializing in diabetic retinal disease and a fluorescein angiogram was obtained. Eye changes were graded as: (a) no retinopathy; $(b)$ background retinopathy (the presence of microaneurysms; or $(c)$ more severe retinopathy, including changes such as retinal hemorrhages, hard exudates, soft exudates, or proliferation of new vessels. 22 of the 26 patients completed a 24-h urine determination for microalbuminuria, which was determined by an RIA (Clinical Assays, Los Angeles, CA). Skin changes were assessed over the dorsum of the fingers and over the forearm by a single observer (B. Buckingham) by palpation and by attempting to tent the skin. Skin changes were graded into three categories: $(a)$ normal; $(b)$ mild but definite thickening; or $(c)$ more severe thickening associated with skin tight- ness. This method of clinical assessment has been used in previous studies of skin changes in diabetes $(12,13)$ and has been shown to correlate with skin core biopsy weight and total dermal collagen in systemic sclerosis (14). At the time of biopsy, each patient was also assessed for flexion contractures of the proximal interphalangeal joints (PIP) of fingers 2-5 by having the patient approximate his fingers in a prayer position; each PIP joint was also assessed with a goniometer. Glycohemoglobin levels were determined by affinity column chromatography (Endocrine Sciences, Tarzana, CA). All diabetic subjects had glycohemoglobin levels measured at the time of biopsy; in addition, most had levels determined $3,6,9,12$, and 15 mo before biopsy.

Biochemical analyses. Skin samples (2-3 mg wet weight) were minced into fine pieces, washed, and reduced with $\mathrm{NaB}^{3} \mathrm{H}_{4}(142 \mathrm{Ci} /$ mol; Amersham Corp., Arlington Heights, IL) as described by us previously (15). The effective reducing capacity of each batch of $\mathrm{NaB}^{3} \mathrm{H}_{4}$ was determined using $\partial$-amino levulinic acid as a standard compound for reduction (16), thus allowing us to determine molar quantities of cross-links from their radioactivity. Reduced tissues were hydrolyzed in $6 \mathrm{~N} \mathrm{HCl}$ and hydroxyproline content was determined colorimetrically (16). The difunctional crosslinks dihydroxylysinonorleucine (DHLNL) and hydroxylysinonorleucine (HLNL), as well as nonenzymatically glycosylated lysine and hydroxylysine, were analyzed by HPLC using an isocratic solvent as eluant, a procedure we have described in detail previously $(15,16)$. Fig. 2 shows a typical chromatograph of a reduced skin hydrolysate. Preparation and characterization of DHLNL and HLNL standards have been described previously (15). We have also prepared and characterized ${ }^{14} \mathrm{C}$-labeled standards for nonenzymatically glycosylated lysine and hydroxylysine by the method of Robins (10). Briefly, equimolar amounts of glucose and radiolabeled lysine ( $100 \mathrm{mCi} / \mathrm{mol}$; Amersham Corp.) were incubated in the presence of $\mathrm{NaCNBH}_{3}$ for $24 \mathrm{~h}$; glycosylated lysine was separated from unreacted lysine with a boronate afinity column (17). This procedure yields radiolabeled alpha and epsilon glycosylated lysine, which are separated by HPLC using our difunctional cross-link analy- 


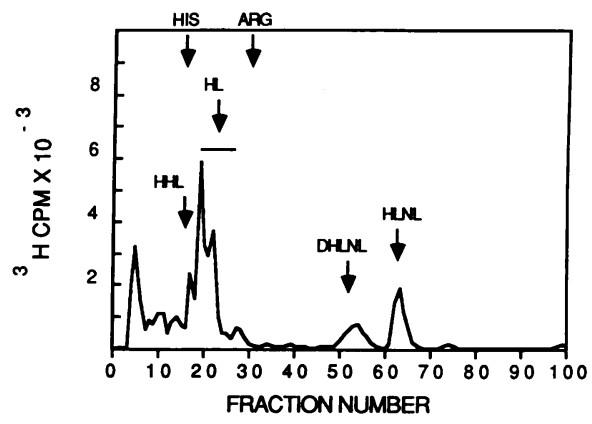

Figure 2. Radiochromatograph of reduced skin hydrolysate, containing $\sim 10 \mu \mathrm{g}$ of hydroxyproline, showing the elution positions of hexosyl-hydroxylysine (HHL), hexosyl-lysine and its hydrolysis rearrangement products (HL), and the difunctional cross-links DHLNL and HLNL. The elution positions of histidine and arginine are indicated by arrows. Column, ultrasphere $\mathrm{C} 18$ reverse-phase, $15 \times 25$ $\mathrm{mm}$. Mobile phase, $0.01 \mathrm{M}$ Na phosphate, $0.3 \%$ SDS, $24 \% n$-propanol, pH 2.8. Flow rate, $1 \mathrm{ml} / \mathrm{min}$. Fractions were collected at 1 -min intervals and counted by liquid scintillation counting.

sis program (15). To confirm the identity of the epsilon glycosylated compound, unlabeled epsilon-glycosylated lysine was prepared by incubating poly(L-lysine) (Pierce Chemical Co., Rockford, IL) with glucose followed by hydrolysis (10) and boronate affinity column chromatography. Further confirmation of the identity of the radiolabeled marker was obtained by subjecting an aliquot to a Smith degradation as described by Robins (10); all of the radioactivity coeluted with authentic lysine. Markers for glycosylated hydroxylysine were prepared by incubating $\left[{ }^{14} \mathrm{C}\right]$ glucose and hydroxylysine in the presence of $\mathrm{NaCNBH}_{3}$ as described above. To confirm that the ${ }^{3} \mathrm{H}$-labeled compounds in skin that coeluted with the ${ }^{14} \mathrm{C}$-labeled marker represented only glycosylated lysine and not contaminating coelutants, an aliquot of reduced skin hydrolysate was chromatographed on a boronate affinity column; the only radioactive peaks present in the eluate coeluted with the ${ }^{14} \mathrm{C}$-labeled markers.

The trifunctional fluorescent cross-links lysyl pyridinium (LP) and hydroxypyridinium (OHP) were analyzed by a modification of the method of Eyre et al. (18). Standards were prepared as described elsewhere (16). Samples containing $5 \mu \mathrm{g}$ of hydroxyproline were chromatographed on a $0.4 \times 25 \mathrm{~cm} \mathrm{C18}$ reverse-phase column (Ultrasphere; Altex, Berkeley, CA), with an elution solvent of $18 \%$ acetonitrile in $0.01 \mathrm{M}$ heptafluorobutyric acid. A fluorometer (model 2000, Hitachi Ltd., Tokyo, Japan) was used for detection (excitation $=295$ $\mathrm{nm}$, emission $=395 \mathrm{~nm}$ ). Samples were also analyzed for the presence of histidinohydroxymerodesmosine (HHMD); histidine-HLNL (hisHLNL), a recently described trifunctional, nonreducible cross-link (19); and PA, a nonfluorescent deoxy analogue of hydroxypyridinium (20). Preparation of an HHMD standard has been described by us previously (21). A standard of known concentration of his-HLNL was generously provided by Dr. Gerald Mechanic (School of Dental Research, University of North Carolina, Chapel Hill, NC). We prepared a partially purified standard of PA from mature bovine skin. It was identified by its elution position relative to all the other cross-links, its predominance in mature skin, and its size as determined by molecular sieve chromatography (20). Samples containing 10-20 $\mu \mathrm{g}$ of hydroxyproline were chromatographed on a $0.25 \times 10 \mathrm{~cm} \mathrm{C18} \mathrm{reversed-phase}$ column, and eluted over 55 minutes with a gradient of $40-60 \%$ acetonitrile in water, $0.15 \% \mathrm{Na}$ dodecylsulfate, $\mathrm{pH}$ adjusted to 1.5 with trifluoroacetic acid, a modification of the method of Tilson et al. (20). All three cross-links (HHMD, his-HLNL, and PA) were detected as their fluorescent adducts after post-column derivatization with $o$ phthalaldehyde (OPA) as previously described (15). HHMD was also detected and quantified by its radioactivity.

Insufficient material was available in the skin biopsies to perform analyses of collagen-linked fluorescence (as an index of advanced
Maillard product content) in addition to the other assays described above.

Statistical analysis. All biochemical studies were done without knowledge of clinical findings or whether the biopsies were from nondiabetic or diabetic subjects. Clinical assessments were done without knowledge of biochemical data. Fluorescein angiograms were read without knowledge of biochemical or clinical data. Statistical analyses were performed using the Biomedical Programs (22) for correlation between two variables (6D), comparison of two groups with $t$ tests (3D), analysis of two-way and multiway frequency tables (4F), and stepwise regression (2R). Tau $\beta$ values were calculated using the Statistical Package for the Social Sciences (SPSS) (23).

\section{Results}

Biochemical assays. We found significant differences in lysyl oxidase-dependent cross-linking and in nonenzymatic glycosylation between diabetic and control skin collagen; the data are summarized in Table I. Diabetics had significantly increased levels of the difunctional cross-link DHLNL with normal levels of HLNL; thus DHLNL:HLNL ratios were also increased. OHP, a trifunctional maturational product of DHLNL and hydroxylysine, was also significantly increased in diabetic skin. OHP was present in 20 of 21 diabetic skin biopsies; however, only 2 of 9 nondiabetics, both 13-yr-old adolescents, had detectable levels of this collagen cross-link. LP was not detected in either control or diabetic skin. PA, a recently described nonfluorescent deoxy analogue of OHP that is present in normal skin (20), was not significantly increased in diabetic skin collagen. The content of his-HLNL and HHMD, two other collagen crosslinks present in human skin, was normal in diabetic skin collagen. The content of nonenzymatically glycosylated lysine (hex-lys) and hydroxylysine (hex-hyl) was significantly increased in diabetic skin collagen; the ratio of hex-hyl to hex-lys did not differ between control and diabetic subjects.

The relationship between DHLNL content and OHP content was of particular interest, as DHLNL is the immediate

Table I. Collagen Content of Lysyl Oxidase-dependent Cross-Links and Nonenzymatically Glycosylated Lysine and Hydroxylysine in Skin Collagen from Control and Diabetic Patients

\begin{tabular}{lcc}
\hline \multicolumn{1}{c}{ Crosslink } & \multicolumn{1}{c}{ Control* $^{*}$} & \multicolumn{1}{c}{ Diabetic } \\
\hline DHLNL & $0.021 \pm 0.016(9)$ & $0.096 \pm 0.058(26)^{\ddagger}$ \\
HLNL & $0.187 \pm 0.030(9)$ & $0.204 \pm 0.023(26)$ \\
DHLNL/HLNL & $0.098 \pm 0.020(9)$ & $0.619 \pm 0.094(26)^{\ddagger}$ \\
His-HLNL & $0.109 \pm 0.023(8)$ & $0.151 \pm 0.024(19)$ \\
HHMD & $0.531 \pm 0.086(8)$ & $0.469 \pm 0.064(17)$ \\
OHP & $0.004 \pm 0.002(9)$ & $0.023 \pm 0.004(17)^{\S}$ \\
PA & $4.914 \pm 0.084(8)$ & $7.028 \pm 0.083(19)$ \\
Hex-lys & $0.292 \pm 0.081(9)$ & $1.126 \pm 0.146(26)^{\ddagger}$ \\
Hex-hyl & $0.054 \pm 0.017(9)$ & $0.181 \pm 0.026(26)^{\prime \prime}$
\end{tabular}

* All data are expressed as moles of cross-link/moles of collagen, with the exception of PA, which is expressed as arbitrary fluorescence units per mol of collagen. The data are presented as mean $\pm S E M$, with the number of samples analyzed in parentheses. Significance was determined using two-tailed $t$ tests without assuming equal variances of the populations. ${ }^{\ddagger} P<0.00001 ;{ }^{\S} P<0.001 ; " P<0.0001$. 
Table II. Correlation of Nonenzymatic Glycosylation of Collagen with Lysyl Oxidase-derived Collagen Cross-Links

\begin{tabular}{lccccc}
\hline & \multicolumn{2}{c}{ Hexosyl-lysine* } & & \multicolumn{2}{c}{ Hexosyl-hydroxylysine } \\
\cline { 2 - 3 } \cline { 6 - 6 } $\begin{array}{l}\text { Cross-link } \\
\text { analyzed }\end{array}$ & IDDM & Control & & IDDM & Control \\
\hline DHLNL & $0.79^{\ddagger}$ & 0.56 & & $0.80^{\ddagger}$ & 0.48 \\
HLNL & 0.45 & $0.80^{\S}$ & & 0.33 & $0.74^{\prime \prime}$ \\
OHP & 0.42 & -0.18 & & $0.54^{\| \prime}$ & -0.29 \\
PA & -0.25 & $-0.73^{\| \prime}$ & & $-0.46^{\| \prime}$ & $-0.75^{\| \prime}$ \\
His-HLNL & 0.15 & 0.59 & & 0.06 & 0.38 \\
HHMD & $0.49^{\| \prime}$ & -0.12 & & 0.39 & -0.09 \\
& & & & & \\
\hline
\end{tabular}

* Relationships between nonenzymatically glycosylated lysine and hydroxylysine residues and lysyl oxidase-derived cross-links are expressed as Pearson correlation coefficients.

${ }^{\ddagger} P<0.001 ;{ }^{\S} P<0.01 ;{ }^{\prime \prime} P<0.05$.

precursor of OHP. We found a strong correlation between DHLNL and OHP values $(r=0.84, P<0.001)$.

We next examined if correlations existed between nonenzymatic glycosylation and lysyl oxidase-dependent crosslinks (Table II). In diabetic subjects, increases in hex-lys and hexhyl were strongly correlated with increased DHLNL levels, as shown in Fig. 3. In nondiabetic subjects, increased hex-lys and hex-hyl were correlated with increased HLNL and decreased PA.

Clinical correlations. In nondiabetic subjects age was not correlated with collagen cross-link content with the exception that his-HLNL increased with age $(r=0.79, P<0.02)$. Fig. 4 shows the relationship between age and DHLNL, OHP, and PA in both control and diabetic subjects. In diabetic subjects,

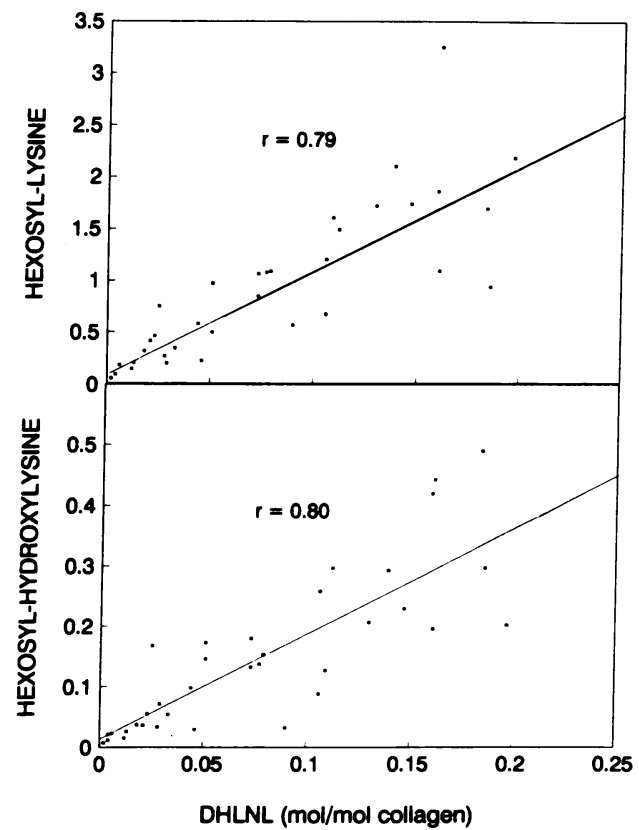

Figure 3. Scattergrams illustrating the correlation between nonenzymatic glycosylation of collagen (hexosyl-lysine and hexosyl-hydroxylysine) and DHLNL in diabetic subjects. Both correlations were significant at $P<0.001$.

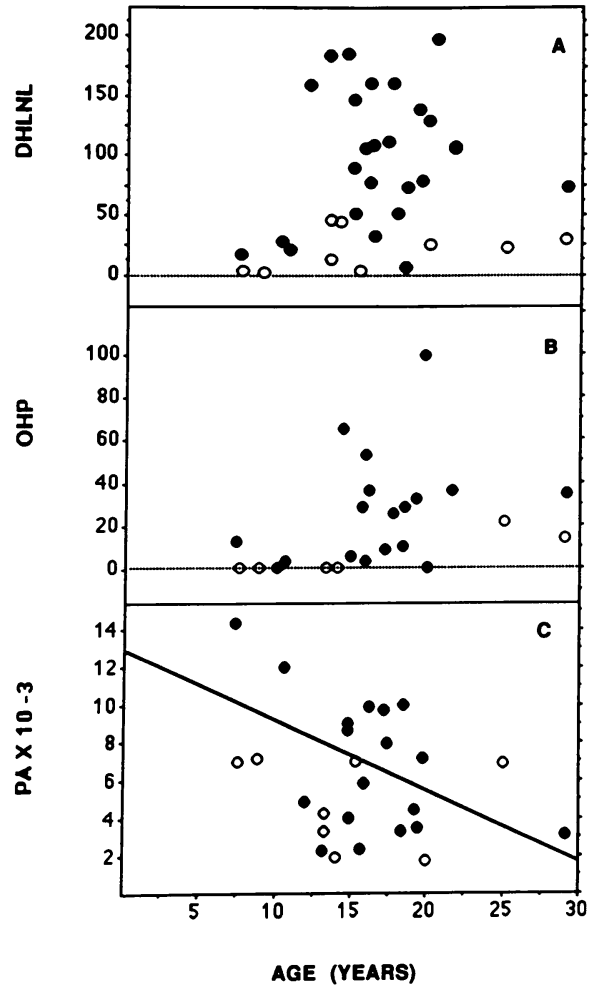

Figure 4. Scattergrams illustrating the relationship between age of control and diabetic subjects and skin content of DHLNL $(A)$, OHP $(B)$, and PA $(C)$. Data are expressed as millimoles of cross-link/ moles of collagen in the case of DHLNL and OHP, and as arbitrary fluorescence units/mol of collagen in the case of PA. A significant correlation $(r=0.51, P<0.02)$ between age and PA content in diabetic subjects was observed; no other correlations were significant. (Open circles) Control subjects; (closed circles) diabetic subjects.

PA levels decreased with age $(r=0.51, P<0.02)$. There were no other correlations between cross-link content and age in diabetic subjects. With increased duration of diabetes, DHLNL content was increased $(r=0.55, P<0.003)$ and OHP was increased $(r=0.59, P<0.01)$, whereas PA levels were decreased $(r=-0.48, P<0.04)$ (Fig. 5). Nonenzymatic glycosylation of collagen was also increased with increased duration of diabetes (hex-lys, $r=0.47, P<0.02$; hex-hyl, $r=0.39, P$ $<0.05$ ).

Glycohemoglobin levels measured at the time of biopsy were correlated with hex-lys, hex-hyl, DHLNL, DHLNL: HLNL and OHP. DHLNL content and the ratio of DHLNL to HLNL were correlated with glycohemoglobin levels obtained up to 15 mo before biopsy (Table III, Fig. 6).

Long-term complications were associated with changes in lysyl oxidase-dependent cross-linking. Analysis of these relationships used Pearson product correlations for continuous variables, such as microalbuminuria, (Table IV) and Kendall's Tau $\beta$ values for noncontinuous variables, such as the presence or absence of background retinopathy (Table VI).

Microalbuminuria was positively correlated with increased levels of hex-lys, hex-hyl, and DHLNL (Fig. 7), as well as with the ratio of DHLNL to HLNL (Table IV). When the effect of age was factored out by using stepwise regression (Table IV), all correlations of microalbuminuria with collagen cross-links 


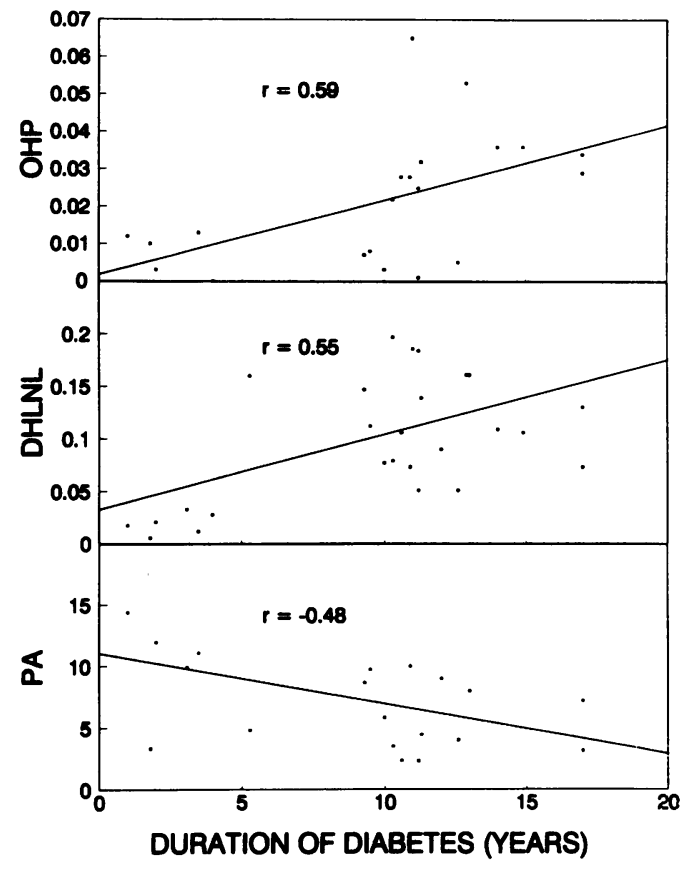

Figure 5. Scattergrams illustrating the correlation between duration of diabetes (years) and skin collagen content of the cross-links OHP, DHLNL, and PA. Values for crosslinks are expressed as moles of cross-link/moles of collagen.

and nonenzymatic glycosylation remained significant. When the effect of diabetes duration was factored out, the correlation of microalbuminuria with nonenzymatic glycosylation persisted, but the correlation with DHLNL and with the DHLNL/HLNL ratio was no longer significant. The signifcant correlation between microalbuminuria and HHMD remained after the effect of diabetes duration was factored out. The patient with the highest microalbuminuria value $(120$ $\mathrm{mg} / \mathrm{g}$ creatinine) was retested $6 \mathrm{mo}$ after the initial value was obtained to determine if this high value was valid. The second test revealed a value of $136 \mathrm{mg} / \mathrm{g}$, suggesting that the initial measurement was biologically valid. If data obtained from this patient are excluded, significant correlations exist between al-

Table III. Correlation of Glycohemoglobin Levels Measured up to 15 mo before Biopsy with Skin Collagen Content of Nonenzymatically Glycosylated Lysine and Hydroxylysine and with DHLNL and DHLNL/HLNL Ratios

\begin{tabular}{ccccc}
\hline Time* & Hex-hyl & Hex-lys & DHLNL & DHLNL/HLNL \\
\hline $0(26)$ & $0.45^{\ddagger}$ & $0.51^{\S}$ & $0.62^{\prime \prime}$ & $0.59^{\prime \prime}$ \\
$3(19)$ & 0.05 & 0.23 & 0.39 & $0.62^{\S}$ \\
$6(21)$ & 0.09 & 0.32 & 0.34 & $0.47^{\ddagger}$ \\
$9(20)^{*}$ & $0.37^{\ddagger}$ & $0.51^{\ddagger}$ & $0.56^{\S}$ & $0.71^{\prime \prime}$ \\
$12(22)$ & $0.49^{\ddagger}$ & $0.68^{\prime \prime}$ & $0.49^{\ddagger}$ & 0.37 \\
$15(10)$ & 0.33 & 0.29 & $0.63^{\ddagger}$ & $0.64^{\ddagger}$
\end{tabular}

* Time refers to months prior to biopsy; the number of subjects is shown in parentheses. Relationships between glycohemoglobin levels and skin content of hex-hyl, hex-lys, and DHLNL and the ratio of DHLNL/HLNL are expressed as Pearson correlation coefficients. ${ }^{\ddagger} P<0.05 ;{ }^{8} P<0.01 ; "$ " $P<0.001$.

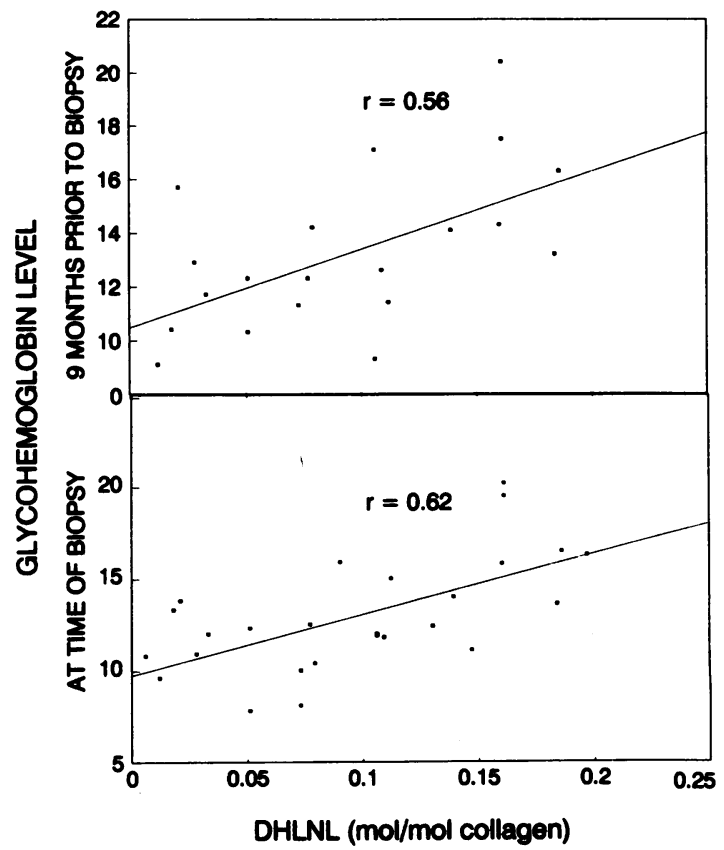

Figure 6. Scattergram illustrating the correlation between glycohemoglobin levels and skin collagen content of DHLNL. The lower scattergram shows glycohemoglobin levels at the time of biopsy and the upper scattergram shows levels obtained 9 mo before the biopsy.

buminuria and DHLNL and hexosyl hydroxylysine content, but not between albuminuria and HHMD, DHLNL:HLNL and hexosyl lysine content.

Four diabetic subjects had contractures of their fifth finger, and eight had multiple joint involvement. Clinical assessment of hand contractures was associated with increased DHLNL/ HLNL ratios $\left(\chi^{2}=7.77, P<0.005\right)$. Goniometer measurements of fifth finger proximal interphalangeal contractures were correlated with increased DHLNL levels and increased

Table IV. Correlation of Microalbuminuria with Collagen Cross-Links and Nonenzymatic Glycosylation of Collagen

\begin{tabular}{lccc}
\hline \multicolumn{1}{c}{ Assay } & Correlation* & $\begin{array}{c}\text { Partial correlation } \\
\text { controlling for age }\end{array}$ & $\begin{array}{c}\text { Partial correlation } \\
\text { controlling for } \\
\text { duration of DM }\end{array}$ \\
\hline DHLNL & $0.49^{\ddagger}$ & $0.49^{\ddagger}$ & 0.31 \\
HLNL & -0.07 & & \\
DHLNL/HLNL & $0.43^{\S}$ & $0.43^{\S}$ & 0.28 \\
OHP & 0.22 & & \\
PA & -0.36 & & \\
HHMD & $0.63^{\ddagger}$ & $0.63^{\ddagger}$ & $0.63^{\ddagger}$ \\
his-HLNL & 0.12 & & \\
Hex-lys & $0.65^{\prime \prime}$ & $0.65^{\prime \prime}$ & \\
Hex-hyl & $0.66^{\prime \prime}$ & $0.66^{\prime \prime}$ & $0.55^{\prime \prime}$ \\
\hline
\end{tabular}

Microalbuminuria ranged from 1 to $120 \mathrm{mg} / \mathrm{g}$ of creatinine in a 24-h urine collection.

* Correlations are expressed as Pearson correlation coefficients. Partial correlations, determined using stepwise regression, were obtained only for those biochemical assays in which a significant correlation was observed with microalbuminuria.

${ }^{\ddagger} P<0.01 ;{ }^{\S} P<0.05 ; "$ " $P<0.001$. 


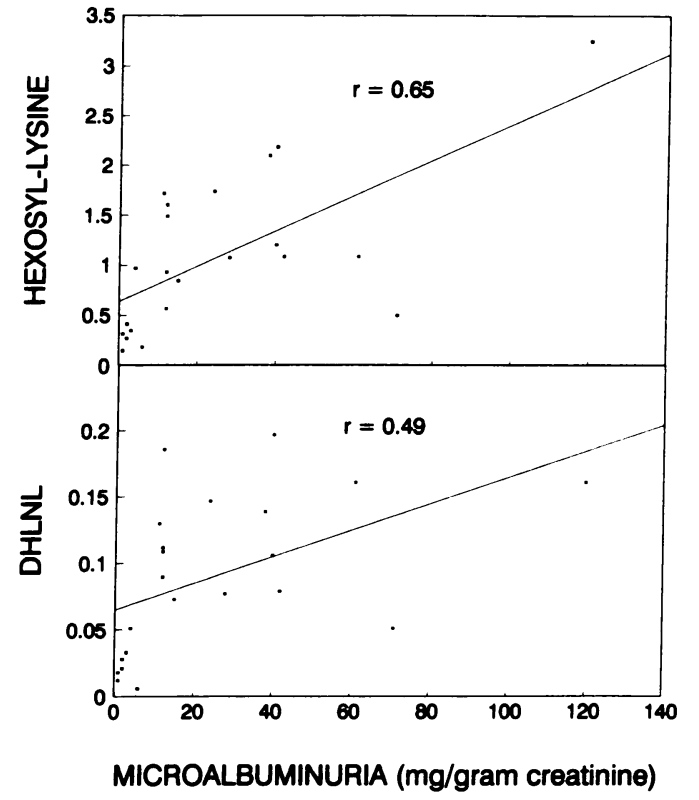

Figure 7. Scattergram showing the correlation between degree of microalbuminuria and skin collagen content of DHLNL and nonenzymatically glycosylated lysine in diabetic subjects. Values for DHLNL and hexosyl-lysine are expressed as moles/moles of collagen.

OHP levels (Table V). When the effect of age was factored out by stepwise regression, the correlations of contractures with DHLNL and OHP content were still significant, but when the effect of diabetes duration was factored out, these correlations were no longer significant.

Retinal findings were limited to the presence of microaneurysms in six subjects and no subject had retinal hemorrhages, hard or soft exudates, or new vessel formation. The presence of background retinopathy in 6 of the 25 diabetic subjects was associated with increased DHLNL, DHLNL/HLNL ratios,

Table V. Correlation of Contractures with Collagen Cross-Links and with Nonenzymatic Glycosylation of Collagen

\begin{tabular}{lccc}
\hline \multicolumn{1}{c}{ Assay } & Correlation* $^{*}$ & $\begin{array}{c}\text { Partial correlation } \\
\text { controlling for age }\end{array}$ & $\begin{array}{c}\text { Partial correlation } \\
\text { controlling for } \\
\text { duration of DM }\end{array}$ \\
\hline DHLNL & $0.39^{\ddagger}$ & $0.39^{\ddagger}$ & 0.18 \\
HLNL & -0.15 & & \\
DHLNL/HLNL & 0.32 & & \\
OHP & $0.70^{\S}$ & $0.51^{\prime \prime}$ & 0.36 \\
PA & -0.05 & & \\
HHMD & 0.25 & & \\
His-HLNL & 0.06 & & \\
Hex-lys & 0.32 & & \\
Hex-hyl & 0.27 & & \\
& & & \\
\hline
\end{tabular}

The amount of contracture of the right fifth finger ranged from 0 to 16 degrees by goniometer assessment.

* Correlations are expressed as Pearson correlation coefficients. Partial correlations, determined using stepwise regression, were obtained only for those biochemical assays in which a significant correlation was observed with joint changes.

${ }^{\ddagger} P<0.05 ;{ }^{\S} P<0.002$; " $P<0.02$. decreased HLNL content, and increased OHP content (Table VI).

Clinical assessment of skin changes over the dorsum of the fingers revealed that 6 diabetics had no skin involvement, 5 had thickened skin, and 15 had both thickening and tightness of their skin. On clinical assessment of skin changes over the forearm, 10 patients had no skin changes, 7 had thickening of their skin and 9 had both thickening and tightness of their skin. Thickening and tightness of the skin were associated with increased DHLNL content, an increased DHLNL/HLNL ratio, increased OHP, and increased nonenzymatic glycosylation of collagen (Table VI). Skin changes over the fingers and forearm were associated with the presence of joint contractures $\left(\chi^{2}=8.22, P<0.02\right.$; and $\chi^{2}=12.99, P<0.003$, respectively). Skin changes were not significantly correlated with the degree of microalbuminuria or the presence of background retinopathy. 2 of 13 patients with microalbuminuria values of $<22$ $\mathrm{mg} / \mathrm{g}$ of creatinine had retinopathy and 4 of 9 patients with increased microalbuminuria had retinopathy $(P=N S)$. Four patients, two of whom had retinopathy, failed to complete a 24-h urine collection.

\section{Discussion}

This study was designed to test three hypotheses: first, that lysyl oxidase-dependent cross-linking is increased in skin collagen in IDDM; second, that changes in nonenzymatic glycosylation would correlate with changes in collagen crosslinks; third, that changes in crosslink content could be correlated with clinical complications. Our data suggest that lysyl oxidase-dependent cross-linking is indeed increased in IDDM: Diabetic patients had significantly increased content of DHLNL and OHP in their skin collagen as compared with age-matched controls. Particularly intriguing is the fact that the cross-links affected in diabetes appear to arise from one specific pathway: DHLNL and its products (see Fig. $1 B$ ). The content of all of the other cross-links surveyed in this study were normal, including HLNL, its trifunctional product hisHLNL, and HHMD, a tetrafunctional cross-link arising from the condensation of two lysine-derived aldehydes. To our

Table VI. Results of Frequency Table Analysis of Clinical Data with Skin Collagen Content of Lysyl Oxidase-derived Cross-Links and with Hexosyl Lysine and Hexosyl-Hydroxylysine

\begin{tabular}{|c|c|c|c|}
\hline Assay & Retinopathy* & $\begin{array}{l}\text { Skin changes } \\
\text { (hand) }\end{array}$ & $\begin{array}{l}\text { Skin changes } \\
\quad \text { (arm) }\end{array}$ \\
\hline DHLNL & $0.30^{\ddagger}$ & $0.40^{\S}$ & $0.37^{\S}$ \\
\hline HLNL & $-0.44^{\S}$ & 0.15 & 0.16 \\
\hline DHLNL/HLNL & $0.59^{\prime \prime}$ & $0.48^{\prime \prime}$ & $0.36^{\prime}$ \\
\hline OHP & $0.42^{\pi}$ & $0.39^{\top}$ & 0.23 \\
\hline PA & -0.04 & -0.09 & -0.16 \\
\hline HHMD & -0.04 & -0.05 & 0.14 \\
\hline His-HLNL & 0.08 & 0.24 & 0.27 \\
\hline Hex-lys & 0.17 & $0.36^{\pi}$ & $0.38^{\S}$ \\
\hline Hex-hyl & 0.08 & $0.39^{\S}$ & $0.44^{\prime \prime}$ \\
\hline
\end{tabular}

* Data are expressed as Kendall Tau- $\beta$ values. Retinopathy categories: $(a)$ none, $(b)$ background. Skin change categories: $(a)$ normal, (b) tight, $(c)$ thick and tight.

${ }^{\ddagger} P<0.05 ;{ }^{\S} P<0.005 ; "$ " $P<0.001 ;{ }^{~} P<0.01$. 
knowledge, this is the first study in which specific lysyl oxidase-dependent cross-links have been assayed in tissue from patients with IDDM. The observation that the increase in lysyl oxidase-dependent cross-links in diabetes is restricted to DHLNL and its maturation product OHP suggests that the cross-linking abnormalities cannot be solely attributed to an increase in lysyl oxidase activity. Rather, this observation suggests that changes in the extent of lysine hydroxylation, an intracellular modification of collagen mediated by the enzyme lysyl hydroxylase, may also be an important alteration of collagen metabolism in diabetic subjects.

To our knowledge, only two earlier studies have been done in which lysyl oxidase-mediated cross-links were specifically quantified in experimental diabetes. LePape et al. (24) found no increases in either HLNL or HHMD content of rat tail tendon 6 and 12 mo after induction of diabetes with streptozotocin. Similarly, we found no changes in HLNL or HHMD content in diabetic subjects. In a separate study, LePape et al. (25) reported that the ratio of DHLNL to HLNL was increased in glomerular basement membrane (GBM) collagen in experimental diabetes due to a decrease in HLNL content. It should be noted that cross-link content was expressed as radioactivity present in alkaline hydrolysates in the study cited. It is not clear if this is an accurate technique for comparison of crosslink content between tissues without additional information regarding recovery and purity of the preparation. It is also possible that diabetes does not affect type IV collagen crosslinking in the same way that it affects cross-linking in the interstitial collagens, such as type I (the major collagen type in skin).

The changes in lysyl oxidase-dependent cross-links observed in diabetes in this study resemble cross-link abnormalities observed in other connective tissue disorders. For example, specific increases in DHLNL and OHP have been reported in many types of pulmonary fibrosis, hypertrophic scarring, and Dupuytren's contracture (26-31). Of interest in this regard is the increased incidence of Dupuytren's contracture in diabetic patients. The levels of OHP measured in hypertrophic scar collagen are very similar to those we observed in diabetic skin collagen. Interestingly, this disorder appears to be the only other instance in which OHP has been reported in human skin. Of interest, we were able to detect OHP in two pubertal nondiabetic subjects, but not in prepubertal or postpubertal nondiabetic subjects.

We also found significant increases, to more than twice the levels found in controls, in nonenzymatically glycosylated lysine and hydroxylysine residues in diabetic skin collagen. These data are consistent with previous reports of increased nonenzymatic glycosylation of various tissues in both human and experimental diabetes (8). There is as yet no consensus as to how an increase in nonenzymatic glycosylation might affect the properties of collagen. It has been suggested that such an increase is significant primarily because the early glycosylation products are precursors of the advanced Maillard products that are believed to be responsible for the pathogenesis of many of the complications of diabetes (7-9). It was our second hypothesis that an increased extent of nonenzymatic glycosylation of collagen would correlate with changes in collagen crosslinks. We found that increased levels of nonenzymatic glycosylation did indeed correlate with an increased content of DHLNL. It has been suggested that an increase in nonenzymatic glycosylation of collagen could lead to a decrease in lysyl oxidase-dependent cross-linking if sugars competed for the same lysine and hydroxylysine residues that serve as substrates for lysyl oxidase (32). Although not conclusive on this point, our data suggest that such a competition may not be taking place in diabetic subjects, since we found a strong positive correlation between extent of nonenzymatic glycosylation and DHLNL content. It is of interest that in nondiabetic subjects, increase in nonenzymatic glycosylation of collagen was correlated with increases in HLNL, not in DHLNL. It is not clear whether there is a causal relationship underlying these correlations.

The third aim of our study was to examine whether our biochemical data were correlated with clinical findings in the diabetic subjects. We found that nonenzymatic glycosylation of collagen was correlated with glycohemoglobin levels measured at the time of biopsy (and at 9 and 12 mo before the biopsy) and to skin changes, but was not significantly correlated with duration of diabetes, joint stiffness, or background retinopathy. Our data concerning the relationship between nonenzymatic glycosylation and clinical parameters differ slightly from those reported by Vasanth et al. (33) in that we found a weak correlation between extent of nonenzymatic glycosylation and microalbuminuria. This observation may be explained by differences in patient populations in that our patients did not have overt nephropathy $(>500 \mathrm{mg} / \mathrm{g}$ creatinine) and their renal involvement was limited to microalbuminuria ( $<200 \mathrm{mg} / \mathrm{g}$ creatinine).

Increases in DHLNL content or the DHLNL/HLNL ratio were correlated with almost all of the clinical parameters measured. The positive correlation between duration of diabetes and the increase of DHLNL and OHP content suggests that changes in skin collagen may be cumulative, as an increasingly large percentage of skin contains collagen synthesized under "diabetic conditions." Increases in the DHLNL content or the DHLNL/HLNL ratio of skin collagen in diabetic patients were correlated with the presence of background retinopathy, microalbuminuria, and skin and joint changes. These correlations were not dependent on the age of the subject but were dependent on the duration of diabetes. Increases in OHP content of diabetic skin collagen were correlated with retinopathy, skin changes and joint contractures. The relationship between the changes in dermal (presumably types I and III) collagen (skin and joint complications) shown in this study and presumed changes in basement membrane type IV collagen (renal and retinal complications) may be explained by the suggestion that similar biochemical changes are affecting all collagen types in diabetic subjects. Changes in type III collagen might be directly related to changes in diabetic basement membrane collagen since recent studies in diabetic rats have demonstrated that insulin treatment causes expression of type III collagen in the mesangial matrix (34).

The inverse correlation between PA and duration of diabetes deserves comment. It is possible that PA and OHP represent competing biosynthetic pathways in which DHLNL is the common precursor, and in diabetes OHP formation might take precedence. Possibly the increase in nonenzymatic glycosylation of lysine and/or hydroxylysine blocks biosynthesis of PA. Alternatively, PA may not be a maturation product of DHLNL, and any correlations between DHLNL and PA content may simply be associative.

Although we cannot impute causal relationships to our observations, we can speculate on the implications. The corre- 
lation between DHLNL content and glycohemoglobin measurements up to 9 mo before biopsy suggests that long-term glycemic control may play a role in modulating lysyl oxidasedependent cross-linking. Lien et al. (35) suggest that collagen that is synthesized under hyperglycemic conditions will be more susceptible to degradation than normal collagen; hence, diabetic tissue would be selectively depleted of newly synthesized collagen and contain a higher percentage of crosslinked collagen than normal collagen. Such selective depletion could result in collagen with a higher content of DHLNL, a more stable cross-link than HLNL under certain conditions.

Hyperglycemia may also affect intracellular events related to crosslinking. Cagliero et al. (36) have shown that high glucose concentrations lead to an increase in mRNA levels of both type IV collagen and fibronectin in cultured endothelial cells; fibronectin may serve an important regulatory role in the extracellular matrix. Increased lysine hydroxylation has been reported to be increased in hyperglycemic states, and could affect the type of crosslinks synthesized $(37,38)$. Enzymatically mediated glycosylation of collagen may also be associated with hyperglycemia, although there is controversy on this point (37-39). Yamauchi et al. (40) have shown that alterations in enzymatic glycosylation can directly affect the types of crosslinks formed.

It is of interest that several of the biochemical changes described in diabetic skin collagen are similar to changes observed in fetal collagen; i.e., an increase in type III collagen relative to type I collagen $(34,41)$ and, in our present report, an increase in DHLNL. Perhaps some of the hormonal changes associated with diabetes, such as increased insulin and growth hormone levels, stimulate epithelial cells to revert to a pattern of fetal collagen production, with increased lysyl hydroxylase activity and increased type III collagen production. Although it is possible that the alterations in DHLNL observed in diabetic skin could be attributed to an increase in type III collagen, there is evidence that, at least in hypertrophic scarring, increases in DHLNL are associated with type I collagen as well as with type III collagen (29).

Much recent attention has focused on the role of advanced Maillard products in the pathogenesis of diabetic complications. Collagen-linked fluorescence, imputed to the presence of such products, has been shown to be increased in diabetic skin collagen and the increased content is strongly correlated with long-term complications (7-9). Our data are consistent with the hypothesis that Maillard products play a central role in diabetic complications. Indeed, the two processes of collagen crosslinking may be interrelated. It is possible, for example, that abnormal lysyl oxidase-dependent cross-linking results in collagen that turns over more slowly, thus facilitating the further stabilization of these fibrils by Maillard products. Alternatively, the two processes may proceed independently, the net result being the accumulation of physical, chemical, and microscopic abnormalities observed in diabetic collagen.

\section{Acknowledgments}

We thank Dr. Robert Ruper for performing the angiograms and Suzanne Hennessy and Mary Zombek for expert technical assistance. Statistical consultation was provided by Dr. James Ashurst, statistical consultant, Academic Computing, University of California, Irvine.
This work was supported in part by National Institutes of Health grant AG-05324, RR-00169, and HL-32690 and by Pediatric-Adolescent Diabetes Research and Education Foundation (PADRE).

\section{References}

1. Francis, M. J. O., and J. P. Ellis. 1974. Skin collagen in diabetes mellitus in relation to treatment. Proc. $R$. Soc. Med. 67:35-36.

2. Hamlin, C. R., and J. H. Luschin. 1975. Apparent accelerated aging of human collagen in diabetes mellitus. Diabetes. 24:902-904.

3. Golub, L. M., R. A. Greenwald, E. J. Zebrowski, and N. S. Ramamurthy. 1978. The effect of experimental diabetes on the molecular characteristics of soluble rat-tail tendon collagen. Biochim. Biophys. Acta. 534:73-81.

4. Trueb, B., R. Fluckiger, and K. H. Winterhalter. 1984. Nonenzymatic glycosylation of basement membrane collagen in diabetes mellitus. Collagen Relat. Res. 4:239-251.

5. Rosenbloom, A. L., J. H. Silverstein, D. C. Lezotte, K. Richardson, and M. McCallum. 1981. Limited joint mobility in childhood diabetes mellitus indicates increased risk for microvascular disease. $N$. Engl. J. Med. 305:191-194.

6. Buckingham, B. A., J. Uitto, C. Sandborg, T. Keens, T. Roe, G. Costin, F. Kaufman, B. Bernstein, B. Landing, and A. Castellano. 1984. Scleroderma-like changes in insulin-dependent diabetes mellitus: clinical and biochemical studies. Diabetes Care. 7:163-169.

7. Brownlee, M., A. Cerami, and H. Vlassara. 1988. Advanced glycosylation end-products in tissue and the biochemical basis of diabetic complications. N. Engl. J. Med. 318:1315-1322.

8. Kennedy, L., and J. W. Baynes. 1984. Non-enzymatic glycosylation and the chronic complications of diabetes: an overview. Diabetologia. 26:93-98.

9. Monnier, V. M., V. Vishwanat, Frank Kay E., Elmets Craig A., P. Dauchot, and R. R. Kohn. 1986. Relation between complications of type I diabetes mellitus and collagen-linked fluorescence. $N$. Engl. J. Med. 314:403-408.

10. Robins, S. P. 1982. Analysis of the crosslinking components in collagen and elastin. Methods Biochem. Analysis. 28:330-379.

11. Chang, K., J. Uitto, E. A. Rowold, G. A. Grant, C. Kilo, and J. R. Williamson. 1980. Increased collagen cross-linkages in experimental diabetes: reversal by beta-aminoproprionitrile and D-penicillamine. Diabetes. 29:778-781.

12. Siebold, J. R. 1982. Digital sclerosis in children with insulindependent diabetes mellitus. Arthritis Rheum. 25:1357-1360.

13. Buckingham, B., A. J. Perejda, C. Sandborg, A. K. Kershnar, and J. Uitto. 1986. Skin, joint and pulmonary changes in type I diabetes mellitus. Am. J. Diseases Childhood. 140:420-423.

14. Rodnan, G. P., E. Lipinski, and J. Luksick. 1979. Skin thickness and collagen content in progressive systemic sclerosis and localized scleroderma. Arthritis Rheum. 22:130-140.

15. Reiser, K. M., and J. A. Last. 1983. Analysis of collagen composition and biosynthesis by HPLC. Liquid Chromatogr. 1:498-502.

16. Reiser, K. M., S. M. Hennessy, and J. A. Last. 1987. Analysis of age-associated changes in collagen crosslinking in the skin and lung in monkeys and rats. Biochim. Biophys. Acta. 926:339-348.

17. Yue, D. K., S. McLennan, L. Delbridge, D. J. Handelsman, T Reeve, and J. R. Turtle. 1983. The thermal stability of collagen in diabetic rats: correlation with severity of diabetes and nonenzymatic glycosylation. Diabetologia. 24:282-285.

18. Eyre, D. R., T. J. Koob, and K. P. Van Ness. 1984. Quantitation of hydroxypyridinium crosslinks in collagen by high-performance liquid chromatography. Anal. Biochem. 137:380-388.

19. Yamauchi, M., R. E. London, C. Guenat, F. Hashimoto, and G. L. Mechanic. 1987. Structure and formation of a stable histidinebased trifunctional cross-link in skin collagen. J. Biol. Chem. 24:11428-11434.

20. Tilson, M. D., R. N. Dreyer, A. Hudson, R. J. Cotter, and M. L. Tanzer. 1985. Biochem. Biophys. Res. Commun. 126:1222-1227.

21. Reiser, K. M., W. S. Tyler, S. M. Hennessy, J. J. Dominguez, 
and J. A. Last. 1987. Long-term consequences of exposure to ozone: II. Structural alterations in lung collagen of monkeys. Tox. Appl. Pharmacol. 89:314-322.

22. Dixon, W. J. 1981. BMDP Statistical Software. University of California Press, Berkeley, CA.

23. Nie, N. H., C. H. Hull, J. G. Jenkins, H. Steinbrenner, and D. H. Bent. 1975. Statistical Package for the Social Sciences. McGraw-Hill Book Co., New York.

24. LePape, A., J. Muh, and A. J. Bailey. 1981. Characterization of $\mathrm{N}$-glycosylated type I collagen in streptozotocin-induced diabetes. Biochem. J. 197:405-412.

25. LePape, A., J. D. Guitton, N. Gutman, Y. Legrand, F. Fauvel, and J. P. Muh. 1983. Nonenzymatic glycosylation of collagen in diabetes: incidence on increased normal platelet aggregation. Haemostasis. 13:36-41.

26. Reiser, K. M., F. Tryka, R. C. Lindenschmidt, J. A. Last, and H. R. Witschi. 1986. Changes in collagen cross-linking in bleomycininduced pulmonary fibrosis. J. Biochem. Toxicol. 1:83-91.

27. Reiser, K. M., and J. A. Last. 1986. Collagen crosslinking in lungs of rats with experimental silicosis. Collagen Relat. Res. 6:313324.

28. Last, J. A., A. Siefkin, and K. M. Reiser. 1983. Type I collagen is increased in lungs of patients with adult respiratory distress syndrome. Thorax. 38:364-368.

29. Bailey, A. J., S. Bazin, T. J. Sims, M. L. Lous, C. Nicoletis, and A. Delaunay. 1975. Characterization of the collagen of human hypertrophic and normal scars. Biochim. Biophys. Acta. 405:412-421.

30. Moriguchi, T., and D. Fujimoto. 1979. Crosslink of collagen in hypertrophic scar. J. Invest. Dermatol. 72:143-145.

31. Brickley-Parsons, D., M. J. Glimcher, R. J. Smith, R. Albin, and J. P. Adams. 1981. Biochemical changes in the collagen of the palmar fascia in patients with Dupuytren's disease. J. Bone Joint Surg. 5:787-797.

32. Li, W., S. Shen, G. A. Robertson, M. Khatami, and J. H.
Rockey. 1984. Increased solubility of newly synthesized collagen in retinal capillary pericyte cultures by nonenzymatic glycosylation. $O p h$ thalmic Res. 16:315-321.

33. Vasanth, V., K. E. Frank, C. A. Elmets, P. J. Dauchot, and V. M. Monnier. 1986. Glycation of skin collagen in type I diabetes mellitus. Diabetes. 35:916-921.

34. Abrass, C. K., C. V. Peterson, and G. J. Raugi. 1988. Phenotypic expression of collagen types in mesangial matrix of diabetic and nondiabetic rats. Diabetes. 37:1695-1702.

35. Lien, Y., R. F. Stern, and R. C. Siegel. 1984. Inhibition of collagen fibril formation in vitro and subsequent cross-linking by glucose. Science (Wash. DC). 225:1489-1491.

36. Cagliero, E. M., and M. Lorenzi. 1988. High glucose increases collagen type IV and fibronectin messenger RNAs in cultured human endothelial cells. Clin. Res. 36:478a. (Abstr.)

37. Romen, W., H. W. Lange, K. Hempel, and T. Heck. 1981. Studies on collagen metabolism in rats: II. Turnover and amino acid composition of the collagen of glomerular basement membrane in diabetes mellitus. Virchows Arch. Cell. Pathol. 36:313-320.

38. Beisswenger, P. J., and R. G. Spiro. 1972. Studies on the human glomerular basement membrane: composition, nature of the carbohydrate units and chemical changes in diabetes mellitus. Diabetes. 22:180-193.

39. Kefalides, N. A. 1974. Biochemical properties of human glomerular basement membrane in normal and diabetic kidneys. J. Clin. Invest. 53:403-407.

40. Yamauchi, M., C. Noyes, Y. Kuboki, and G. L. Mechanic. 1982. Collagen structural microheterogeneity and a possible role for glycosylated hydroxylysine in type I collagen. Proc. Natl. Acad. Sci. USA. 79:76784-7688.

41. Kern, P., B. Sebert, and L. Robert. 1986. Increased type III/type I collagen ratios in diabetic human conjunctival biopsies. Clin. Physiol. Biochem. 4:113-119. 